\title{
Asociaciones de inmigrantes mexicanos en Estados Unidos: logros y desafíos en tiempos recientes*
}

\author{
LUIS ESCALA-RABADÁN
}

En el contexto del creciente debate público y político sobre la migración de mexicanos hacia Estados Unidos, debido principalmente a su alta densidad, permanencia y vasta distribución, ha habido también una creciente atención pública, política y académica hacia las organizaciones comunitarias formadas por estos inmigrantes. Este artículo examina el desarrollo y la paulatina centralidad de asociaciones y federaciones de migrantes mexicanos en Estados Unidos, basadas en el lugar o región de origen en México. El texto evalúa su desarrollo inicial y la importancia de sus logros en ambos países durante las últimas décadas, así como los retos que enfrentan en su futuro desarrollo organizativo.

PALABRAS CLAVE: inmigración mexicana en Estados Unidos, asociaciones de inmigrantes mexicanos, mexicanos en Los Ángeles y Chicago, transnacionalismo entre México y Estados Unidos, retos organizativos de asociaciones inmigrantes

Mexican Immigrant Associations in the United States: Challenges and Achievements in Current Times

In a context characterized by the public and political debate on Mexican immigration to the United States, as a result of its high density, settlement, and wide distribution, there has also been an increasing public, political, and academic interest on community organizations forged by immigrants. This article examines the rise and growing importance of these associations and federations of Mexican immigrants in the United States, based on their hometowns in Mexico. The paper assesses their initial development and their significant achievements in both nations during the last decades, as well as some of the key organizational challenges they face in their future.

KEYWORDS: Mexican immigration in the United States, Mexican immigrant associations, Mexicans in Los Angeles and Chicago, transnationalism between Mexico and the United States, organizational challenges among immigrant associations 


\section{Introducción}

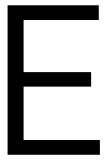

n fechas recientes el debate sobre inmigración en Estados Unidos ha ganado una considerable centralidad en los ámbitos público, político y académico, lo que da continuidad a las posiciones de respaldo y de crítica al papel de los inmigrantes en ese país. Entre los primeros hay actores sociales que apoyan la presencia de los migrantes en nombre de sus contribuciones a la sociedad y la economía, o de sus derechos humanos. Entre los segundos hay también participantes de las esferas política, mediática e incluso académica que sostienen una crítica de moderada a radical hacia la presencia inmigrante, pero en particular hacia los indocumentados provenientes de México. Esta discusión tiene una mediana historia, con posiciones y contextos cambiantes, y no suele incluir un adecuado balance sobre la complejidad y las contribuciones que han hecho las comunidades inmigrantes. Si bien la construcción de la migración como una amenaza a la sociedad estadounidense es claramente una distorsión, lo mismo podría decirse de las representaciones que hacen sus defensores al señalar a estas comunidades de inmigrantes meramente como víctimas pasivas de la opresión y la discriminación en Estados Unidos.

Después de décadas de movimiento continuo, los inmigrantes mexicanos han establecido extensas redes migratorias entre sus lugares de origen en México y de destino en Estados Unidos. En contraste con las imágenes forjadas en los imaginarios públicos y políticos de dicha inmigración como un fenómeno creciente y caótico, la consolidación de estos circuitos migratorios ha hecho posible la acción colectiva de comunidades entre ambas naciones con impactos en sus lugares de origen, de destino o en ambos. Este conjunto de vínculos y redes, forjado por los inmigrantes mexicanos y no muy conocido en la esfera pública y política de Estados Unidos, es lo que se identifica cada vez más como una "sociedad civil migrante", que en términos generales comprende diversos sectores, pero sobre todo se basa en las formas asociativas de las que se ha dotado durante muchos años. 
Las asociaciones conformadas por los inmigrantes mexicanos en Estados Unidos poseen una larga historia, pero se puede apreciar una nueva oleada desde los años ochenta del siglo pasado, tanto en áreas metropolitanas como en zonas rurales, lo mismo en destinos clásicos de la migración mexicana que en nuevos lugares de llegada. Esta tradición asociativa ha adoptado diversas formas: organizaciones religiosas, políticas, deportivas y de promoción cultural. ${ }^{1}$ No obstante, es probable que las asociaciones basadas en el pueblo de origen hayan ganado una creciente atención de los sectores académico, mediático y político desde la década de 1990 a partir de su labor filantrópica y su participación en esquemas de cooperación con los gobiernos de México para la promoción de desarrollo local. Estas asociaciones - conocidas con diversos nombres: clubes de oriundos, comités cívicos o asociaciones de pueblos, y en la literatura anglófona de ciencias sociales denominadas hometown associations (HTA) - y las federaciones que las agrupan han ganado visibilidad de manera paulatina y se han convertido en una forma asociativa adoptada por inmigrantes de diversos países de origen en Estados Unidos, pero sobre todo de México.

La presencia creciente de estas asociaciones revela al menos dos cambios importantes en el perfil de las formas asociativas de los inmigrantes mexicanos: hacia su interior y hacia el exterior. El primero consiste en que dichos grupos han dado pasos considerables en la consolidación de sus formas organizativas. En contraste con la relativa informalidad y la fragmentación de las asociaciones de este tipo en años previos, estas asociaciones presentan un perfil renovado, con un número ascendente de ellas agrupadas en federaciones y confederaciones, que se han convertido en actores sociales cada vez más formales y visibles en ambos lados de la frontera, con capacidad de interlocución e incidencia en diversos ámbitos. El segundo cambio se refiere a la creación paulatina de vínculos con otros grupos y actores políticos y sociales, más allá de los lazos que solían tejerse sólo con sus lugares de origen.

La densa red de relaciones que han logrado establecer estas agrupaciones de inmigrantes entre sus comunidades de origen y de llegada ha sido la base para hablar de comunidades y procesos transnacionales, tanto en la academia como en los ámbitos político y mediático. A su vez, esto ha permitido tener una mejor comprensión de las asociaciones basadas en la localidad o región de origen. En ese sentido, estos grupos junto a otros tipos de organizaciones -laborales, políticas, deportivas, religiosas o étnicas - han logrado ampliar la visibilidad e importancia de la dimensión asociativa de la migración mexicana en Estados Unidos.

En este artículo examino las características generales del proceso de consolidación de estas asociaciones inmigrantes y los cambios centrales en su funcionamiento durante los últimos años. Hago un recuento sobre la dimensión asociativa de la migración inmigrante mexicana en Estados Unidos e identifico algunos procesos fundamentales en la configuración organizativa de estos grupos. Para ello utilizaré buena parte de la literatura que ha tomado como referentes principales a las asociaciones y federaciones de este tipo en las regiones metropolitanas de Los Ángeles y Chicago, que han constituido escenarios clave para el surgimiento y consolidación de estos grupos. En la primera parte presento una reseña sobre la trayectoria y características generales de estas asociaciones. En la segunda analizo algunos de los logros centrales de dichos grupos, como parte de su consolidación como asociaciones inmigrantes en Estados Unidos,

1 En su investigación sobre trayectorias de integración de los migrantes mexicanos en la región de Los Ángeles, Alarcón, Escala-Rabadán y Odgers (2012) detectaron seis formas asociativas: basadas en el lugar de origen, relacionadas con cuestiones educativas, religiosas, culturales, deportivas y de negocios y oficios. 


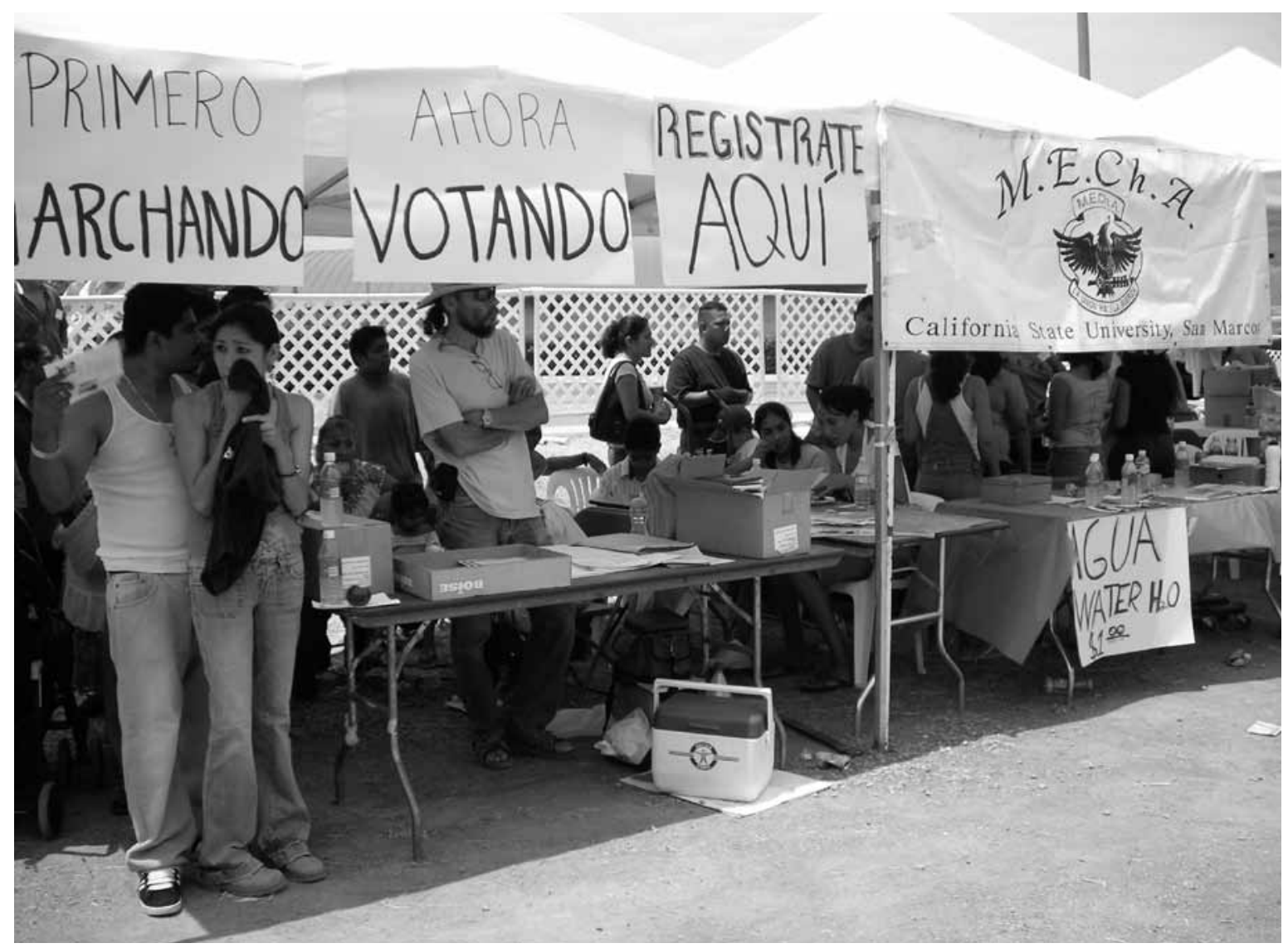

LuIS EsCALA-RABAdÁN • Movimiento Estudiantil Chicano de Aztlán (MECHA) durante la Guelaguetza organizada por la Coalición de Comunidades Indígenas de Oaxaca (Cocio) en la ciudad de San Marcos del condado de San Diego, California, 2006.

en particular su participación cívica y política a nivel binacional. Por último, expongo algunos de los retos que enfrentan dichas asociaciones en el proceso de su consolidación como forma asociativa inmigrante.

\section{Primera parte: trayectorias y características de las asociaciones de inmigrantes mexicanos en Estados Unidos}

Los inmigrantes mexicanos poseen una larga historia de participación cívica y social a través de su acción colectiva, ya sea en sus comunidades de origen, en sus lugares de llegada o bien en ambos espacios, lo que nos permite hablar de "comunidades transnacionales". Ciertamente, este concepto ha sido objeto de diversas críticas y redefiniciones en tiempos recientes. ${ }^{2}$ No obstante, autores como Fox (2005a, 2005b, 2005c) han subrayado la importancia de identificar las prácticas de "binacionalidad cívica" inmersas en estas redes sociales que se establecen entre dos o más naciones. Dichas prácticas de "transnacionalismo" en los estudios de las migraciones, véase Waldinger (2013). 
son parte de lo que se ha denominado "sociedad civil migrante", que constituyen la base de las organizaciones de los propios inmigrantes (Fox, 2005a: 4).

Si bien existen diversos componentes que integran dicha sociedad civil migrante, las organizaciones formadas por y para los propios inmigrantes constituyen su fundamento básico. Estos grupos se basan en identidades colectivas que a veces se traslapan: etnicidad, trabajo, religión o lugar de origen. Sobre todo este último elemento, el paisanaje, es el vínculo que sustenta a las asociaciones conocidas como "clubes de migrantes" y a sus federaciones con sede en sus estados de origen en México, que paulatinamente han ganado centralidad en el universo asociativo de la inmigración mexicana en Estados Unidos. Desde luego, el vínculo de oriundez o paisanaje ha sido una constante de las formas asociativas a lo largo de la historia de las migraciones. ${ }^{3}$ Sin embargo, para el caso que nos atañe es importante examinar precisamente su desarrollo y consolidación como tales, y con ello poder evaluar sus logros como parte de esta "binacionalidad cívica".

La consolidación sucesiva de redes sociales entre regiones específicas de México y Estados Unidos permitió el surgimiento de asociaciones de paisanos basadas en la localidad o región de origen. Esta forma asociativa constituye una versión más formal de la vasta gama de redes informales establecidas por los inmigrantes mexicanos a partir de la pertenencia al mismo pueblo o región de origen en México. ${ }^{4}$ Se advierte su gradual proliferación, con altas y bajas, durante el siglo Xx, aunque con una presencia cada vez más visible desde la década de 1960, con membresías de diversas magnitudes y niveles de organización. ${ }^{5}$

Un elemento fundamental en el surgimiento y desarrollo de estas asociaciones es el fortalecimiento de vínculos entre los inmigrantes y sus pueblos en México, lo que transforma a dichas localidades en referentes importantes para la creación de una identidad colectiva entre los inmigrantes de una misma comunidad o región. Esta conexión se convierte en una parte esencial de su organización social, análoga a los vínculos entre familiares y amigos. Estos vínculos con "la patria chica", lejos de atenuarse o desaparecer con la distancia, se fortalecen y se transforman en redes que eventualmente conducen a la conformación de asociaciones como mecanismos privilegiados de pertenencia "translocal". En este contexto, organizarse en Estados Unidos les permite a los inmigrantes promover y consolidar un sentimiento de identidad cultural compartida. ${ }^{6} \mathrm{La}$ presencia y visibilidad de estas asociaciones de inmigrantes mexicanos osciló en el transcurso del siglo $\mathrm{xx}$ de acuerdo con las condiciones imperantes y la capacidad organizativa de las comunidades inmigrantes. Sin embargo, durante las tres últimas décadas aproximadamente esta presencia ha sido más

3

Véanse, por ejemplo, los trabajos de Soyer (1997) y Moya (2005).

$4 \quad$ Para una revisión de la evolución histórica de estas asociaciones, véase Díaz, Orozco, y González (1997). Existe una amplia literatura sobre la importancia de las redes sociales en el proceso migratorio entre México y Estados Unidos, por ejemplo: Massey et al. (1987), Mines (1981), Zabin et al. (1993) y Light (2006).

5 Para un análisis más detallado sobre el surgimiento de estas asociaciones migrantes en Estados Unidos desde diversas perspectivas, véanse Bada (2003a), Espinosa (1999), Goldring (2002), Rivera (2003), Rivera-Salgado (1999) y Smith (2001), entre otros.

$6 \quad$ La importancia de las asociaciones de migrantes mexicanos en la construcción de una identidad local o nacional puede apreciarse también a través de la evidencia histórica. Por ejemplo, y de acuerdo con algunos analistas, ya existían grupos de mexicanos en California durante la segunda mitad del siglo xix para la promoción de celebraciones de festejos nacionales. De esta forma, fomentaron un sentido de conciencia étnica entre los mexicanos en Estados Unidos. Dicha tradición organizativa se mantuvo durante las décadas del siglo xx, por ejemplo el Club Independencia en Los Ángeles o la Confederación de Sociedades Mexicanas de los Estados Unidos de América en Chicago en 1925 (García, 1996; Taylor, 1997). Sobre la formación de la vasta red de clubes floresmagonistas en Los Ángeles durante la Revolución Mexicana y su influencia en ambos lados de la frontera, véase el trabajo de Vázquez Valenzuela (2012). 
evidente. Un claro indicador de la importancia adquirida por esta forma asociativa es su crecimiento estable en años recientes, como se ilustra en el cuadro 1.

Si bien estos datos son limitados y deben tomarse con cautela, puesto que se basan en el número de asociaciones registradas por la red de consulados mexicanos en Estados Unidos, que habitualmente presenta muestras visibles de subrepresentación o sobrerrepresentación, es claro que la presencia de este tipo de asociaciones se ha ampliado. En ese sentido, el cuadro 1 muestra que en dicho crecimiento

\begin{tabular}{|c|c|c|c|c|}
\hline \multicolumn{5}{|c|}{$\begin{array}{l}\text { CUADRO 1. CLUBES DE MIGRANTES MEX } \\
\text { EN LOS ESTADOS UNIDOS POR LUGAR D } \\
\text { PERIODOS 1998-2003 Y 2009-2011 }\end{array}$} \\
\hline \multirow{2}{*}{ Estado } & \multicolumn{4}{|c|}{ Número de clubes } \\
\hline & 1998 & 2003 & 2009 & 2011 \\
\hline Jalisco & 74 & 100 & 31 & 93 \\
\hline Michoacán & 19 & 51 & 55 & 122 \\
\hline Zacatecas & 113 & 126 & 12 & 25 \\
\hline Guanajuato & 40 & 48 & 307 & 450 \\
\hline Distrito Federal & 3 & 6 & 6 & 13 \\
\hline Sinaloa & 12 & 17 & 5 & 16 \\
\hline Durango & 19 & 20 & 54 & 75 \\
\hline Nayarit & 22 & 27 & 5 & 10 \\
\hline Guerrero & 23 & 51 & 15 & 64 \\
\hline Puebla & 12 & 34 & 38 & 61 \\
\hline Estado de México & 6 & 11 & 14 & 16 \\
\hline Oaxaca & 22 & 36 & 74 & 74 \\
\hline Hidalgo & 4 & 11 & 101 & 134 \\
\hline Aguascalientes & 3 & 1 & 8 & 13 \\
\hline Otros & 69 & 84 & 667 & 676 \\
\hline Total & 441 & 623 & 1392 & 1842 \\
\hline
\end{tabular}

Fuente: González Zepeda (2012: 54), con base en los datos de Escala-Rabadán, Bada y Rivera-Salgado (2006) y SRE (2009 y 2012). participan asociaciones de inmigrantes provenientes de prácticamente todas las entidades de México, tanto de la región tradicional de emigración mexicana como de nuevas zonas, lo que constata el éxito de estas formas organizativas entre los inmigrantes mexicanos de primera generación en Estados Unidos. Asimismo, los datos también sugieren que el aumento de la población inmigrante mexicana ha ido a la par del incremento de sus asociaciones. De hecho, aunque las membresías pudiesen integrar un número muy reducido comparado con la población inmigrante en general, se trata de una "masa crítica" bien organizada que ha sido fundamental en la promoción de una participación cívica y política entre dicha población.

En la actualidad existen literalmente cientos de estas asociaciones a lo largo y ancho de Estados Unidos. En muchos casos se trata de grupos informales, conocidos sólo por sus integrantes, con escaso contacto con otros grupos, ya sea en México o en Estados Unidos. Sin embargo, esta incipiente forma asociativa suele ser el primer paso para su eventual formalización. Por lo general, sus actividades principales se centran en la organización de eventos para la recaudación de fondos con fines filantrópicos: bailes, rifas, concursos de belleza y otras actividades culturales y sociales cuyas ganancias se destinan a obras e iniciativas en sus pueblos de origen en México. Estos eventos cumplen dos objetivos centrales: por un lado, permiten a las asociaciones financiar sus proyectos en sus localidades en México y, por otro, promueven un sentido de comunidad al fortalecer los nexos entre paisanos. De ahí que la creación de estas asociaciones sea un elemento importante para la consolidación de vínculos entre comunidades mexicanas a ambos lados de la frontera (Goldring, 2002; Rivera-Salgado, 1999; Zabin y Escala-Rabadán, 2002; Ímaz, 2006; Moctezuma, 2011).

El crecimiento de estas formas asociativas entre los inmigrantes mexicanos condujo al eventual surgimiento de federaciones, que aglutinaban a 
dichas asociaciones. Para el caso de Los Ángeles, la primera organización de este tipo fue la Federación de Clubes Mexicanos Unidos, fundada en 1972 con clubes de migrantes mexicanos provenientes de Jalisco, Chihuahua, Michoacán, Guanajuato y Zacatecas, cuyo objetivo era fortalecer los vínculos entre asociaciones de este tipo y apoyarlas en sus proyectos filantrópicos en México. Este modelo organizativo de asociaciones basadas en los pueblos de origen y federaciones que las aglutinaban respaldó el desarrollo de las comunidades inmigrantes mexicanas durante los años setenta y ochenta del siglo pasado, en la medida en que la población migrante iba en aumento y se tornaba más estable, en buena parte como resultado de la amnistía de la Immigration Reform and Control Act (IRCA) de 1986. Durante este periodo, los contactos de dichas asociaciones con las instancias del gobierno mexicano eran esporádicos e informales (Zabin y Escala-Rabadán, 2002).

A mediados de la década de 1980 dichos contactos se incrementaron y de manera paulatina las federaciones se radicaron en las entidades mexicanas de origen para agrupar a clubes de inmigrantes con base en dicho criterio. A partir de ello, las federaciones se convirtieron en interlocutores importantes

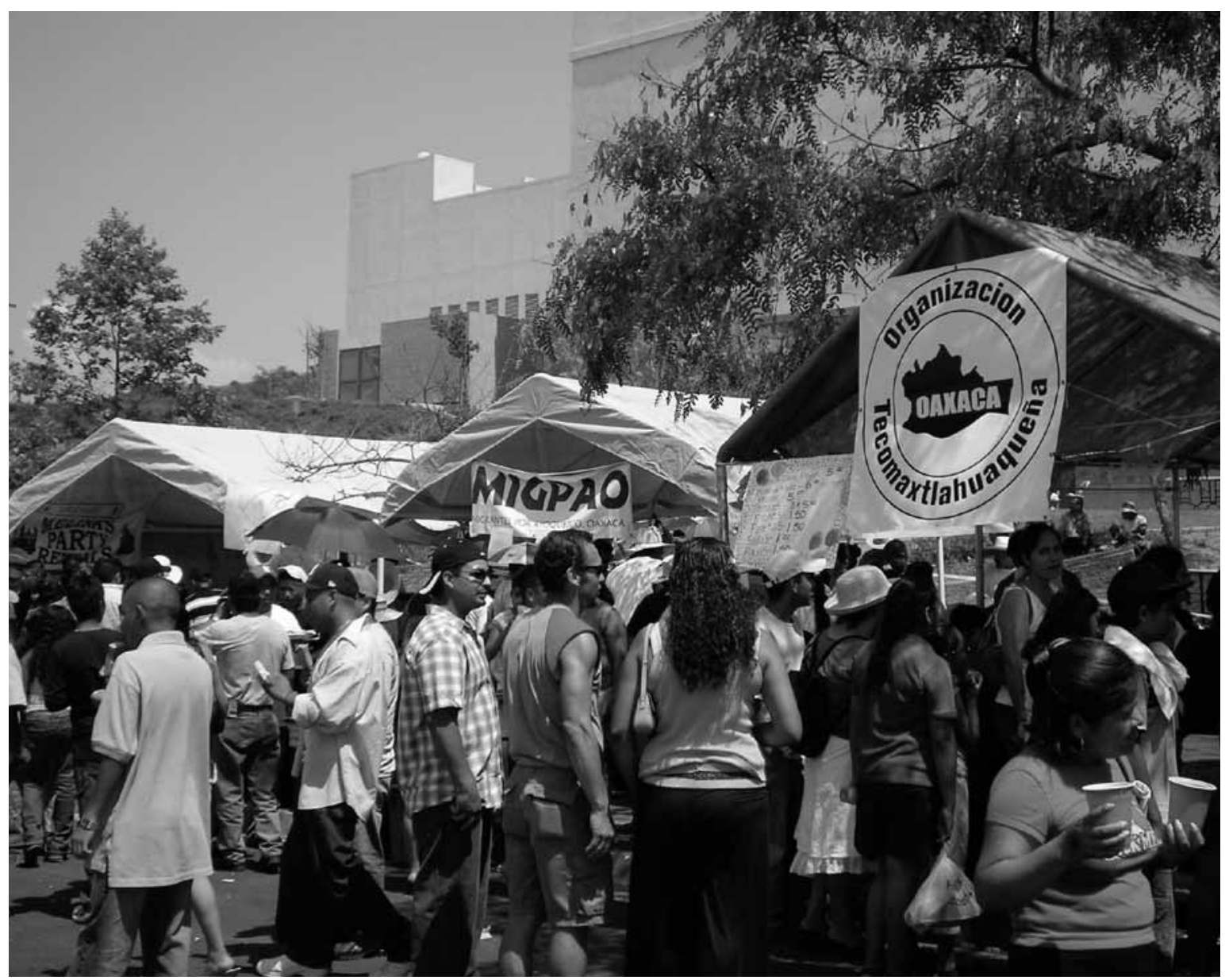

Luis Escala-Rabadán • Asociación Tecomaxtlahuaqueña de San Sebastián, Tecomaxtlahuaca en la Guelaguetza emprendida por Cocio, 2006. 
de las diversas instancias gubernamentales de México, como parte de la diáspora mexicana en Estados Unidos, así como de otros actores sociales y políticos en este país (González Gutiérrez, 1993, 1995; Rivera-Salgado, 1999; Zabin y Escala-Rabadán, 2002; Ímaz, 2006; Moctezuma, 2011). En la década siguiente, como resultado de la creciente presencia de estas asociaciones y de las políticas de acercamiento con las comunidades inmigrantes por parte del gobierno mexicano - en particular el Programa para las Comunidades Mexicanas en el Exterior (PCME) - , los clubes y las federaciones se consolidaron como el modelo organizativo por excelencia entre dichas comunidades. ${ }^{7}$

Al igual que en la región de Los Ángeles, los clubes y federaciones en el medio oeste de Estados Unidos provienen de una larga tradición asociativa entre los inmigrantes mexicanos, como asociaciones mutualistas que apoyaban a los migrantes en situaciones difíciles y en el proceso de su integración a las sociedades receptoras (García, 1996). Durante y después de los años de la Gran Depresión, a fines de la década de 1920, muchas de estas asociaciones desaparecieron como resultado de las deportaciones masivas de inmigrantes mexicanos y de problemas organizativos, pero reaparecerían bajo la forma de clubes y federaciones de inmigrantes durante los años sesenta del mismo siglo (Bada, 2003a).

A mediados de los años noventa del siglo xx el consulado mexicano en Chicago registraba un total de 35 asociaciones de este tipo en el área metropolitana, a finales de esa década llegaban a 140 y para 2005 se estimaba que eran aproximadamente 270, organizadas en 17 federaciones y una Confederación de Federaciones Mexicanas (Confemex) (Barceló, 2005). El crecimiento en esa región forma parte de la expansión de este modelo organizativo entre los inmigrantes mexicanos en Estados Unidos. En la actualidad se calcula que en todo el país hay más de mil asociaciones de este tipo, de acuerdo con el registro de la red de consulados mexicanos
(Gordon, 2005; Vázquez Mota, 2005). Cabe hacer notar que la proliferación de estas asociaciones de la sociedad civil migrante mexicana en las últimas décadas contrasta con el estancamiento de los grupos de la sociedad civil en Estados Unidos, cuyo declive era visible desde mediados de los años setenta del siglo pasado (Putnam, 2000). De hecho, el modelo organizativo de clubes y federaciones de inmigrantes mexicanos se ha expandido en regiones como el medio oeste, donde participan tanto en iniciativas relacionadas con la promoción del desarrollo local en sus lugares de origen en México como en el ámbito cívico y político de su entorno de llegada, por ejemplo, sindicatos locales, asociaciones de vecinos, iglesias o escuelas (Ímaz, 2006; Ramakrishnan y Bloemraad, 2008).

\section{Segunda parte: consolidación y logros de las asociaciones de migrantes mexicanos}

Si bien durante años las asociaciones de inmigrantes mexicanos pasaron inadvertidas tanto para los gobiernos como para los actores sociales y políticos de México y Estados Unidos, su crecimiento, expansión y consolidación las transformó al cabo del tiempo en interlocutores de relevancia. A partir de su visibilidad ascendente, el gobierno mexicano instrumentó nuevas políticas de atención a sus comunidades de inmigrantes para facilitar las relaciones entre comunidades en ambos lados de la frontera, optimizar los recursos materiales y financieros proporcionados por las organizaciones

Buena parte de la literatura sobre clubes y federaciones de inmigrantes mexicanos en Estados Unidos se centra en ciertas regiones. Para el caso de los migrantes zacatecanos, véanse Goldring (1995), Moctezuma (2011) y Mestries (1998); para Jalisco, Morán (1998); para Oaxaca, Fox y Rivera-Salgado (2004), y para clubes de inmigrantes de Michoacán, Espinosa (1999) y Bada (2003b). 
$\mathrm{y}$, desde luego, para generar fuentes adicionales de legitimidad y capital político para los distintos niveles del gobierno de México (Ímaz, 2006; González Gutiérrez, 2006; Fernández, García y Vila, 2006; Viramontes, 2008; Yrízar y Alarcón, 2010). Como resultado, los programas de dichas políticas han permitido no sólo la canalización de recursos de los inmigrantes mexicanos organizados en Estados Unidos hacia sus localidades de origen, las llamadas "remesas colectivas", sino que han creado un mecanismo institucional a través del cual estas asociaciones pueden interactuar con los niveles locales, estatales y federal del gobierno mexicano, con la creciente intermediación de oficinas de atención por parte de las entidades en México, lo que ha dado lugar a una relación unas veces cómoda y otras veces conflictiva entre asociaciones y gobiernos (Ímaz, 2006; Yrízar y Alarcón, 2010).

Aunque la consolidación de esta estructura organizativa es un logro de las propias comunidades inmigrantes mexicanas, lo cierto es que la participación de las políticas gubernamentales de México ha sido crucial en el afianzamiento de los clubes y federaciones como modelo organizativo (Goldring, 1995 y 2002; González Gutiérrez, 2006; Fernández, García y Vila, 2006; Moctezuma, 2011). Casi todas las comunidades inmigrantes mexicanas han adoptado este modelo, probablemente por las ventajas que ofrece en la interacción entre gobiernos y la demanda de las asociaciones de independencia política. Por un lado, las federaciones están mejor posicionadas para interactuar con diversas instancias de México y Estados Unidos, lo que les permite apoyar los objetivos e iniciativas de las asociaciones que aglutinan. Por otro, las diversas instancias del gobierno mexicano encuentran más ventajoso negociar y establecer acuerdos con las federaciones, así como resolver los problemas que pudiesen surgir en la relación entre gobierno y diásporas (EscalaRabadán, 2004; Rivera-Salgado y Escala-Rabadán, 2008; Viramontes, 2008).
Un ejemplo claro de esta relación es la Federación Zacatecana de Los Ángeles, en la medida en que ha logrado establecer una sólida relación con los representantes del gobierno mexicano. Estos vínculos, aunados a la historia organizativa de esta federación, la han convertido en un intermediario fundamental entre las asociaciones que la conforman y las instancias gubernamentales en México (Rivera-Salgado y Escala-Rabadán, 2008; Viramontes, 2008). Los clubes de inmigrantes zacatecanos en California han aprovechado ampliamente esta intermediación efectiva para implementar un gran número de proyectos de desarrollo local en sus comunidades de origen, en comparación con otras federaciones de migrantes mexicanos en Estados Unidos, mediante esquemas de participación como el Programa $3 \times 1$ para Migrantes. ${ }^{8}$

De manera similar a lo que ocurre en Los Ángeles, los clubes y federaciones de inmigrantes mexicanos en el área de Chicago han adquirido visibilidad por medio del despliegue de sus acciones colectivas, centradas en la realización de proyectos para el mejoramiento de las condiciones de vida en sus lugares de origen. En el caso de la región del medio oeste de Estados Unidos, las asociaciones de inmigrantes michoacanos son probablemente las que presentan los logros más destacados en este ámbito. Entre los años setenta y noventa del siglo $\mathrm{xx}$ dichos grupos promovieron un extenso número de proyectos en sus pueblos a través de las remesas colectivas que lograron recaudar y sin apoyo gubernamental. Es hasta fines de la década de 1990 que estas

Prácticamente todos los análisis sobre la Federación y los clubes zacatecanos en Los Ángeles han subrayado los logros obtenidos a través de este programa como un claro indicador de la fortaleza de dichas organizaciones. Además de los trabajos citados, véanse Esparza (2000), Orozco y Welle (2005) y García Zamora (2000, 2001 y 2012). Asimismo, este alcance también ha sido objeto de atención mediática, por ejemplo Thompson (2005) en The New York Times. 
asociaciones inician su participación en el Programa $3 \times 1$ (Bada, 2003b; Bada y Mendoza, 2013). ${ }^{9}$

Uno de los mayores logros de la labor realizada por estas asociaciones ha consistido en canalizar obras y proyectos hacia sus comunidades de origen en México, sobre todo en localidades de marcada marginación. Burgess (2005) ha documentado los resultados de estas acciones, ya que históricamente gran parte de estos recursos se orientaba a las cabeceras municipales de los estados y se desatendían las necesidades de localidades más remotas y vulnerables dentro de un mismo municipio. A través del Programa $3 \times 1$, de acuerdo con esta autora, las asociaciones de inmigrantes michoacanos orientaron $75 \%$ de sus proyectos hacia localidades fuera de la

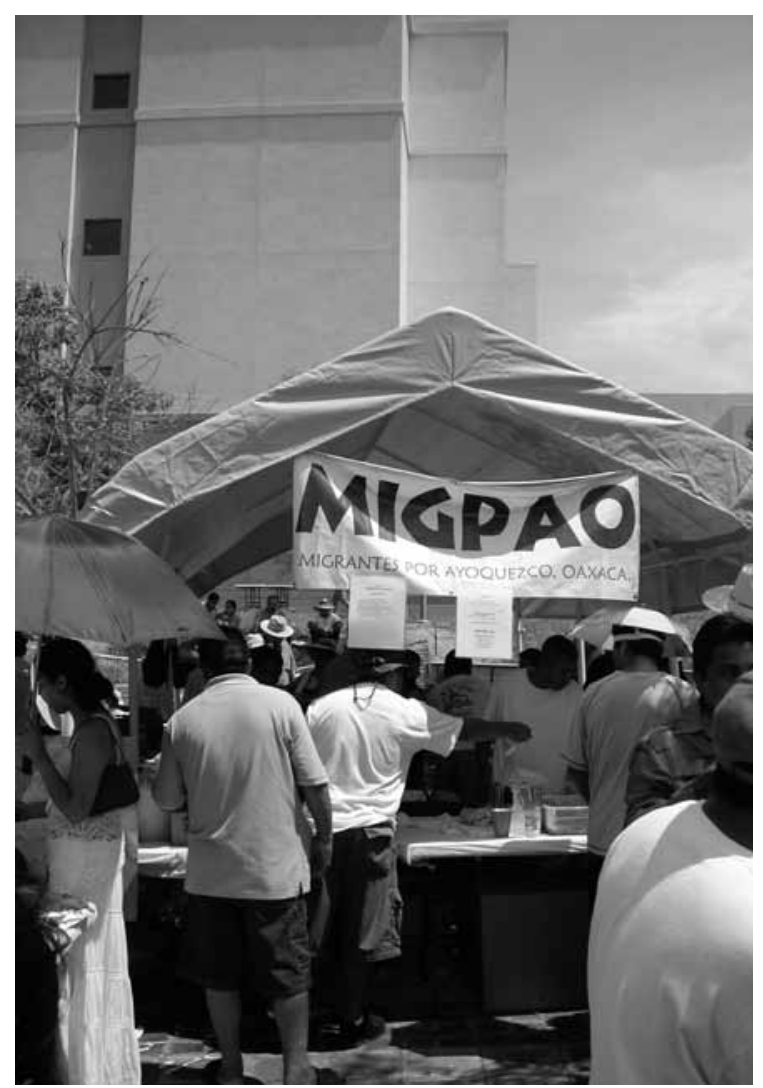

LuIS Escala-Rabadán Puesto de Migrantes por Ayoquezco, Oaxaca (MIGPaO) en el condado de San Diego, California. cabecera municipal, un porcentaje más alto que el de otras federaciones y clubes importantes.

Podría argumentarse que estos programas de cooperación abren la posibilidad de que el gobierno mexicano evada sus responsabilidades en materia de inversión pública para la promoción del desarrollo, sin embargo dichos programas han contribuido de manera sobresaliente al empoderamiento de las comunidades inmigrantes al promover la generación de más y mejores redes, así como a la restauración del tejido social de muchas comunidades de origen en México. De hecho, el financiamiento provisto por los inmigrantes organizados ha permitido la edificación de espacios indispensables para promover mejores condiciones de vida en el México rural, como la reconstrucción de iglesias, plazas públicas, bancas, centros comunitarios y áreas deportivas. Esto es relevante porque en algunos casos la participación cívica puede promoverse sólo a partir de contar con los espacios para discutir las necesidades más apremiantes de las comunidades rurales. Asimismo, los programas de cooperación con el gobierno mexicano en los que participan las asociaciones de migrantes han permitido la promoción de un sentido de pertenencia entre migrantes que con frecuencia experimentan la alienación prevaleciente en las sociedades de llegada. Los proyectos filantrópicos y de infraestructura constituyen un buen motivo para participar y reconectarse con sus lugares de origen (Bada, 2003b; Lanly y Valenzuela, 2004; Moctezuma, 2011; Bada y Mendoza, 2013).

En 2004 el total de la inversión combinada de los tres niveles del gobierno de México y el de los inmigrantes organizados en Estados Unidos alcanzó 66.2 millones de dólares, invertidos en 1263 proyectos. Si bien es una cantidad reducida comparada con el monto de las remesas familiares, tiene mayor potencial para la promoción de formas de desarrollo local más efectivas. La mayoría de dichas remesas colectivas aportadas por las asociaciones de inmigrantes provinieron de California, Illinois y Texas (Vázquez Mota, 2005). 


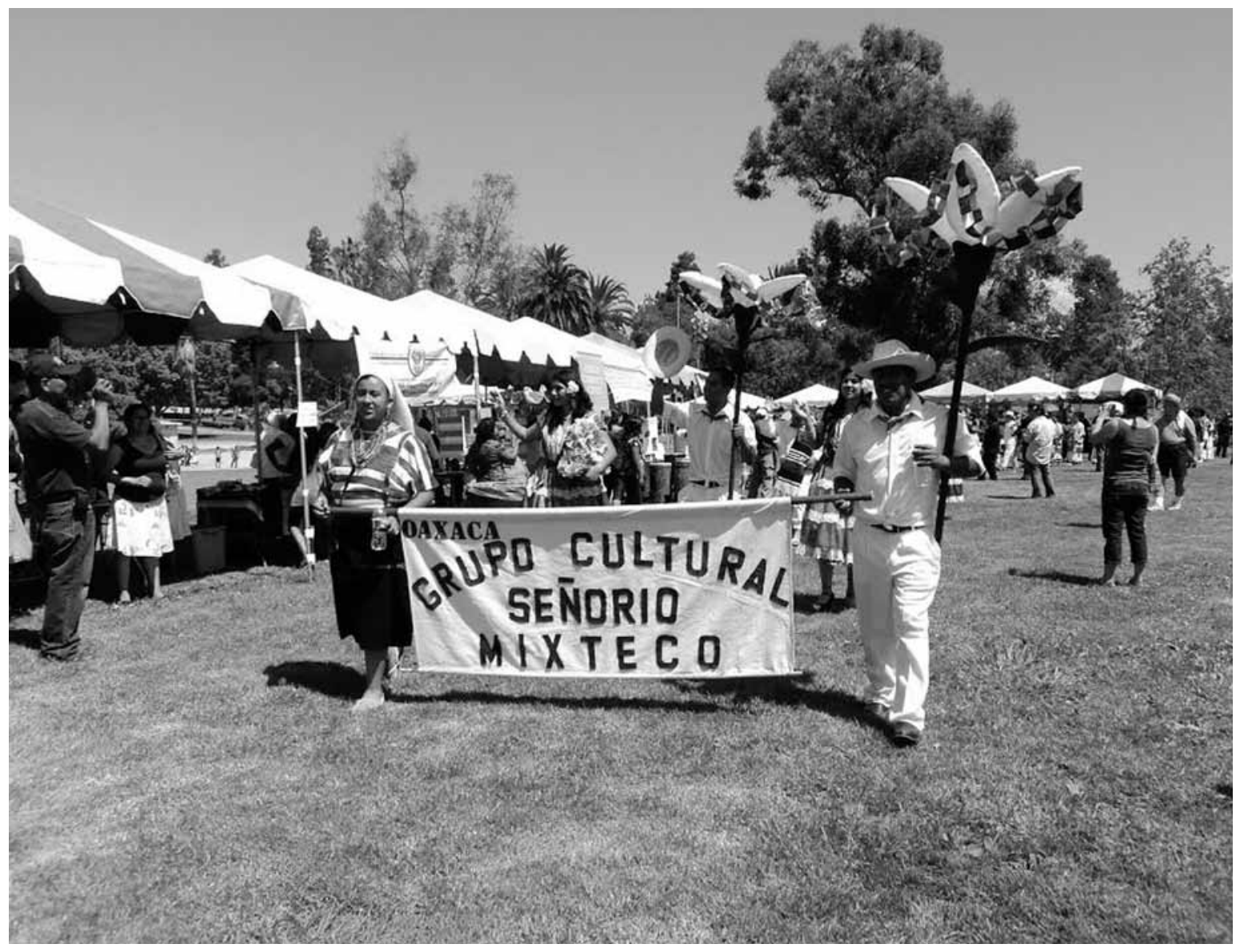

Luis Escala-Rabadán • Grupo de danza "Señorío Mixteco" perteneciente a la Organización Regional de Oaxaca (oro), conformada por migrantes dedicados a promover la cultura dancística oaxaqueña en California, durante la Guelaguetza de 2012 en Los Ángeles, California. Proyecto de investigación sobre Guelaguetzas en California.

La consolidación de esta interlocución progresiva con las dependencias gubernamentales de México ha sido posible gracias al fortalecimiento de las formas asociativas inmigrantes mexicanas, como indica el aumento en el número de clubes, pero también debido a su capacidad para agruparse en federaciones o confederaciones. La mayor visibilidad de estas asociaciones les ha permitido constituirse en voceras de comunidades modestas frente a representantes del gobierno estatal o federal de México para exigir atención y recursos, o para denunciar la corrupción o el autoritarismo de autoridades locales, lo que las erige como posible influencia en la gobernanza local en México (Bada, 2003b; Moctezuma, 2011; Leal y EscalaRabadán, 2011). A su vez, la agenda migrante ha cobrado mayor presencia entre autoridades locales y estatales. Por ejemplo, varios municipios cuentan con representantes en ciudades donde hay concentraciones de inmigrantes originarios de dicha localidad, que se suman a las demandas de maestros, líderes agrarios o sacerdotes de estas localidades (Bada, 2003b). De igual manera, una buena cantidad de estados cuenta con oficinas de atención a sus 
comunidades migrantes en Estados Unidos (Yrízar y Alarcón, 2010).

Otro logro importante es que esta interlocución de las asociaciones de migrantes mexicanos no se restringe a la relación con los distintos niveles de gobierno en México. Su creciente formalización organizativa les ha permitido participar cada vez más en la arena cívica y política de sus entornos de llegada en Estados Unidos. Por ejemplo, durante la primera década del siglo xxI, sus federaciones y confederaciones estuvieron involucradas en varias acciones políticas centradas en los derechos de los inmigrantes (Wides, 2004; Hecht, 2005; Viramontes, 2008; Bada et al., 2010). En contraste con su bajo perfil público en años anteriores, estas asociaciones promovieron vínculos de colaboración con otras organizaciones cívicas y políticas alrededor de la agenda de derechos de los migrantes en Estados Unidos. En 2004 la Confemex fue una de las instancias fundadoras de la National Alliance of Latin American and Caribbean Communities (NALACC), una red de asociaciones de migrantes latinoamericanos y caribeños. En 2005, la prestigiada organización Mexican American Legal Defense and Education Fund (MALDEF) estableció un programa de capacitación para el liderazgo de las asociaciones de inmigrantes mexicanos en California (MALDEF, 2005). A la par de estas relaciones, la Confemex ha participado en iniciativas de cabildeo en regiones metropolitanas como Chicago o Los Ángeles a favor de campañas e iniciativas centradas en el bienestar de las comunidades inmigrantes (Bada et al., 2010).

Si bien esta transformación y consolidación de las formas asociativas de los inmigrantes mexicanos puede explicarse en parte por la dinámica organizativa de los propios inmigrantes y sus comunidades, también es necesario señalar que dichos procesos obedecen a los cambios en el entorno social y político en el que habitan. La marcada densidad de la población inmigrante en Estados Unidos, pero sobre todo su perfil socio-demográfico — son los más jóvenes, con los hogares más grandes, los mayores niveles de pobreza, el menor número de años de residencia, los niveles más bajos de escolaridad y los menores ingresos- y su estatus migratorio, como la mayoría de inmigrantes indocumentados (Passel, 2005), configuran su vulnerabilidad estructural, lo que ha conducido a convertirlos en el blanco del clima antiinmigrante manifiesto en la esfera mediática y política durante la década de 1990 y la primera del siglo xxi (Santa Ana, 2002; Chavez, 2008; EscalaRabadán, Bada y Rivera-Salgado, 2006).

Frente a ello, el movimiento a favor de los derechos de los inmigrantes en Estados Unidos desplegó diversas iniciativas cuyo punto más notorio fue el ciclo de movilizaciones durante 2006 en 158 ciudades, en particular en Chicago, Dallas, Houston y Los Ángeles, que concentran a la mayoría de las asociaciones de inmigrantes mexicanos. Si bien estos inmigrantes participaron en dichas acciones, como parte del presente argumento destaca la visible participación de sus asociaciones. Aunque este movimiento fue organizado inicialmente por un grupo de asociaciones latinas y mexicano-americanas, los clubes y federaciones se sumaron rápidamente (Bada, Fox y Selee, 2006; Viramontes, 2008; Bada et al., 2010). En síntesis, los logros obtenidos por las asociaciones de inmigrantes mexicanos ilustran una transformación de trascendencia en su dinámica organizativa. Tras años de mantener un bajo perfil para gobiernos y actores políticos y sociales en México y Estados Unidos, hoy cuentan con sólidas relaciones con otras asociaciones de inmigrantes, representantes políticos, organizaciones comunitarias, académicos y fundaciones en ambos lados de la frontera. Y si bien esta transformación en su horizonte de acción no es homogénea entre todas las asociaciones de inmigrantes mexicanos ni constituye una evolución lineal y simplista en su dinámica organizativa — de la filantropía enfocada en las comunidades de origen hacia el involucramiento 
cívico en sus lugares de destino-, es importante evidenciar tanto los cambios en sus lugares de llegada como la capacidad de sus líderes y membresías para integrar cada vez más en sus organizaciones una agenda ampliada centrada en los temas de derechos, ciudadanía e inclusión en México y en Estados Unidos.

\section{Tercera parte: desafíos que enfrentan las asociaciones de inmigrantes mexicanos en Estados Unidos}

A pesar de que las asociaciones de inmigrantes mexicanos en Estados Unidos han conseguido importantes conquistas, resultado de su iniciativa y no sólo de las condiciones de sus lugares de llegada o de las políticas de los gobiernos de sus lugares de origen y destino, su eventual desarrollo enfrenta retos, entre los que quisiera señalar los que considero más destacados. El primero, derivado del propio éxito y creciente importancia de las formas asociativas inmigrantes, es la competencia por fungir como representante legítimo de las comunidades inmigrantes mexicanas. Si bien desde la primera década del siglo Xxi las confederaciones de este tipo se posicionaban como legítimas voceras de dichas comunidades, a partir de su extensa membresía provista por las muchas asociaciones que congregaban, otras agrupaciones buscaron adjudicarse dicha representación. ${ }^{10}$

En 2003 el gobierno mexicano creó el Consejo Consultivo del Instituto de los Mexicanos en el Exterior (IME), entidad gubernamental dentro de la Secretaría de Gobernación que comprende un número significativo de representantes inmigrantes de diversas esferas, incluidos los líderes de asociaciones y federaciones (Cano, Molina y Nájar, 2002). El objetivo formal del Consejo es la creación de una vía de enlace entre los inmigrantes mexicanos en Estados Unidos y el gobierno mexicano. No obstante, el reto que enfrentan las asociaciones de migrantes en instancias como ésta consiste en asegurar su autonomía. Aunque el vínculo entre comunidades inmigrantes y el gobierno de México constituye un logro en términos de institucionalización, implica en principio el riesgo de convertirse en una caja de resonancia del gobierno en turno.

El segundo reto ya es una realidad: orientar cada vez más las actividades de las asociaciones hacia el lugar de destino y con ello promover los procesos de integración de sus membresías. Buena parte de estas agrupaciones se caracterizó por dirigir sus acciones casi exclusivamente hacia México y en específico hacia sus localidades de origen, en contraste a una muy limitada vinculación con las sociedades de llegada. Sin embargo, este perfil se ha modificado para dar paso a una presencia progresiva de temas relacionados con las localidades, las ciudades y los estados en los que viven y laboran, además del lugar de origen como referente central, con lo que su naturaleza transnacional se torna más compleja. Las asociaciones parecen desarrollar cada vez más su capacidad de intermediación efectiva en el proceso de promover un sentido de integración social entre sus agremiados al fungir como centros importantes de redes sociales para los nuevos inmigrantes o bien al constituirse como fuente de bienes y servicios, particularmente útiles a sus miembros, como clases de idiomas y el suministro de información y asesoría. La cada vez menor circulación de población migrante entre México y Estados Unidos y una tendencia hacia el establecimiento definitivo delinean un nuevo contexto para las asociaciones de inmigrantes en términos de incluir en sus agendas temas

10

Entre las organizaciones que se decían representantes de los mexicanos en Estados Unidos estaban la Coalición por los Derechos Políticos de los Mexicanos en el Exterior (CDPE), el Frente Cívico Zacatecano (FCZ), la Coalición Internacional de Mexicanos en el Extranjero (CIME) y la Asociación Mundial de Mexicanos en el Exterior (AMME). 


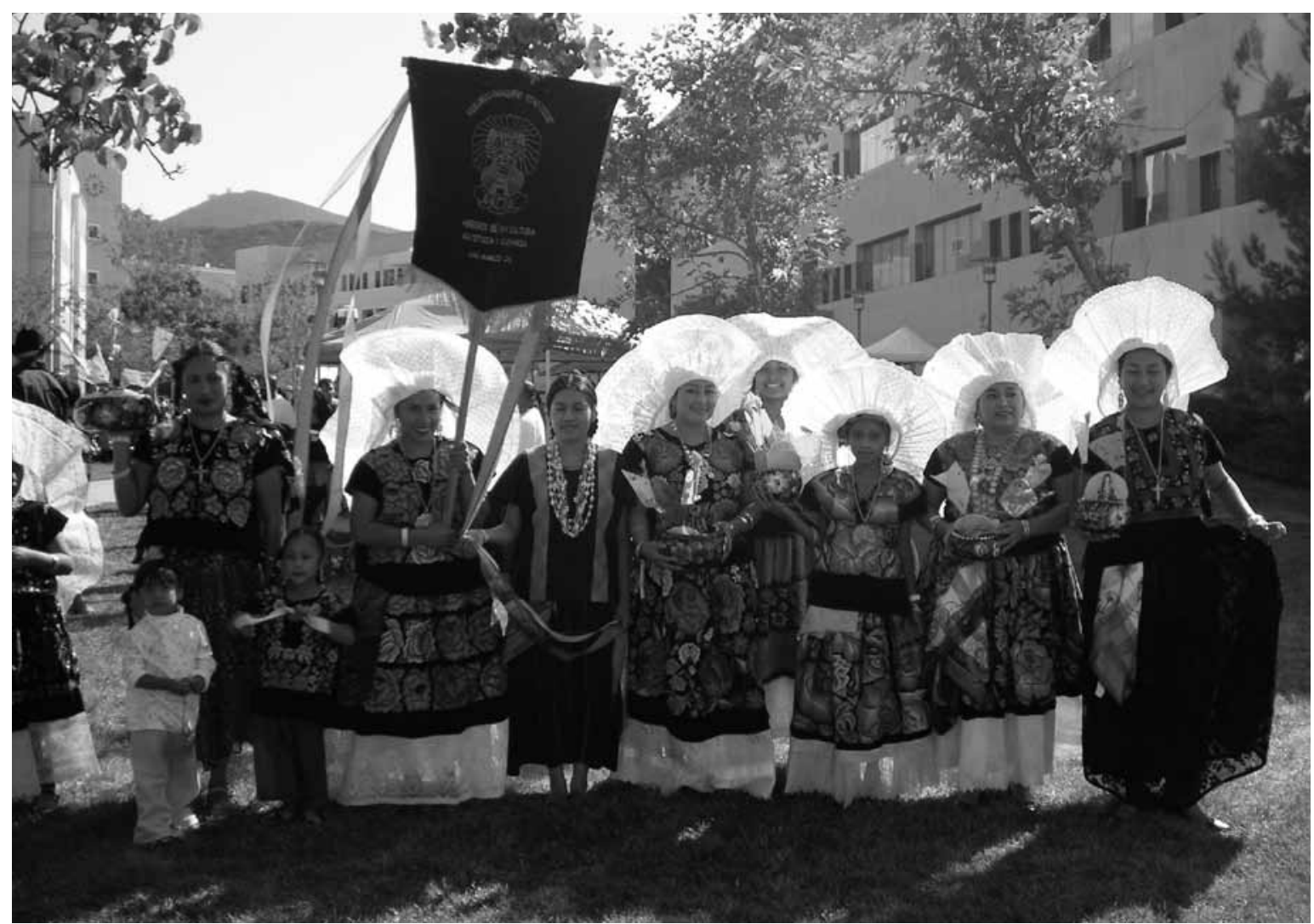

Luis Escala-RabadÁ • Grupo de danza de la Coalición de Comunidades Indígenas de Oaxaca (Cocio) durante la Guelaguetza en San Marcos, California, octubre de 2009.

relativos al futuro de sus miembros y de sus comunidades en los lugares de destino (Alarcón, EscalaRabadán y Odgers, 2012).

Un tercer reto es la promoción de su propio desarrollo organizativo, cada vez más necesario. Una paradoja resultante de los logros mencionados es que con frecuencia su liderazgo se encuentra al límite de su capacidad, como consecuencia de los múltiples compromisos comunitarios, adicionales a los laborales y familiares propios de los inmigrantes mexicanos en Estados Unidos. La visibilidad ascendente de las asociaciones ha llevado al gobierno mexicano y sus políticas de atención a la diáspora, así como a otras instancias sociales y políticas, a alentar su crecimiento numérico y no atender su desarrollo organizativo de manera adecuada, o bien a ofrecerles apoyo y recursos que las agrupaciones no pueden operar por falta de condiciones organizativas, ya que se olvida a menudo que sus integrantes son voluntarios. Varios observadores han advertido este diagnóstico (UNDP, 2006; Shannon, 2006; Somerville, Durana y Terrazas, 2008; Escala-Rabadán, Rivera-Salgado y Rodríguez, 2011) y han señalado la importancia de que el número y la visibilidad de los clubes y federaciones deba acompañarse por la promoción de más y mejores aprendizajes destinados a fortalecer su capacidad organizativa. Ciertamente, varias asociaciones han logrado establecer mecanismos de capacitación con diversas instancias que contribuyen a mejorar su 
funcionamiento como organizaciones. ${ }^{11}$ Sin embargo, también es claro que este desafío debe ser adoptado de manera más decidida en las agendas de las agrupaciones para su desarrollo futuro.

\section{Conclusiones}

He presentado un panorama general sobre las formas asociativas de los inmigrantes mexicanos en Estados Unidos, en particular de los clubes y federaciones con sede en la localidad o región de origen en México. Se incluyó un recuento sobre el origen y el desarrollo de estas asociaciones durante las últimas décadas y después se examinaron los logros obtenidos en su labor organizativa, así como algunos de los principales retos que enfrentan. La existencia de esta densa red de agrupaciones demuestra, en principio, que la migración mexicana en Estados Unidos, lejos de ser un fenómeno masivo y caótico conformado por individuos desposeídos y víctimas pasivas de estructuras omnipresentes, muestra una clara dimensión asociativa de diversos tipos que hace posible la acción colectiva tanto en sus comunidades de origen como en los lugares de destino. De esta manera, la pertenencia a asociaciones radicadas en el pueblo de origen se convierte en un punto de confluencia de varias identidades colectivas y de un eventual sentido de empoderamiento para sus integrantes.

Algunos de los logros obtenidos por estas agrupaciones radican en su consolidación como tales. En contraste con el modesto perfil público en el que vivieron durante años, los clubes y las federaciones de inmigrantes mexicanos cuentan con visibilidad y reconocimiento crecientes por parte de actores sociales y políticos en ambos lados de la frontera. Esto les ha permitido constituirse como interlocutores importantes para la participación en esquemas de cooperación con los distintos niveles del gobierno mexicano, y con ello en la promoción de formas de desarrollo local en sus comunidades de origen. Han participado también en iniciativas relacionadas con los derechos de los inmigrantes en sus lugares de destino, lo que consolida sus niveles de activismo cívico y político. La importancia que dichas asociaciones han adquirido las ha llevado a ampliar su participación en diversas arenas, lo cual ha generado retos para su desarrollo futuro relacionados con su capacidad para mantener su autonomía como organizaciones frente a otros actores, en particular el gobierno de México, y su capacidad para promover su desarrollo organizativo. Desde luego, la respuesta a éstos y otros desafíos es contingente y dependerá de sus liderazgos, sus membresías y sus vínculos con múltiples actores, con el fin de promover más estrategias de participación para sus comunidades tanto en México como en Estados Unidos. Como sugieren Duquette-Rury y Bada (2013), el reto que a su vez enfrentan los académicos frente al cúmulo de información existente sobre las formas asociativas inmigrantes consiste en documentar y analizar con mayor detalle estas transformaciones tanto en su estructura organizativa como en sus lugares de origen y de destino. $\mathbb{D}$

Consúltese, por ejemplo, la experiencia de la Confemex con la Heartland Alliance for Human Needs and Human Rights y con Enlaces América para la promoción de cursos e información entre los miembros de las asociaciones adscritas o la mencionada entre clubes y federaciones de inmigrantes en Los Ángeles con MALDEF, ambas tratadas en EscalaRabadán, Bada y Rivera-Salgado (2006). También el proyecto piloto de formación de capacidades examinado en Escala-Rabadán, Rivera-Salgado y Rodríguez (2011). 


\section{Bibliografía}

Alarcón, Rafael, Luis Escala-Rabadán y Olga Odgers, 2012, Mudando el hogar al norte. Trayectorias de integración de los inmigrantes mexicanos en Los Ángeles, El Colegio de la Frontera Norte, Tijuana.

Bada, Xóchitl, 2003a, Mexican Hometown Associations, Interhemispheric Resource Center (Citizen Action Series in the Americas, núm. 5), Albuquerque.

—_ 2003b, "La participación cívica comunitaria transnacional de los clubes de michoacanos”, en Gustavo López Castro (ed.), Diáspora michoacana, El Colegio de Michoacán, Gobierno del Estado de Michoacán, Zamora.

_- Jonathan Fox y Andrew Selee (comps.), 2006, Invisible No More. Mexican Migrant Civic Participation in the United States, Woodrow Wilson International Center for Scholars, Washington, D. C.

— et al., 2010, La importancia del contexto. El compromiso cívico de los inmigrantes latinos en nueve ciudades de los Estados Unidos, Woodrow Wilson International Center for Scholars, Washington, D. C.

— y Cristóbal Mendoza, 2013, "Estrategias organizativas y prácticas cívicas binacionales de los clubes de oriundos y federaciones mexicanas en Chicago: una perspectiva transnacional desde el /ugar", en Migraciones Internacionales, vol. 7, núm. 1.

Barceló Monroy, Selene, 2005, “La diáspora mexicana y el consulado en Chicago”, en Foreign Affairs en Español, vol. 5, núm. 3.

Burgess, Katrina, 2005, “Migrant Philanthropy and Local Governance in Mexico”, en Barbara Merz (ed.), New Patterns for Mexico: Observations on Remittances, Philanthropic Giving, and Equitable Development, Global Equity Initiative, Harvard University, Cambridge.

Cano, Arturo, Tania Molina y Alberto Nájar, 2002, “El consejo de los migrantes nace bajo fuego. Lista, la representación de los mexicanos en Eu", en Masiosare, 1 de diciembre.

Chavez, Leo R., 2008, The Latino Threat. Constructing Immigrants, Citizens, and the Nation, Stanford University Press, Stanford.

Díaz de Cossío, Roger, Graciela Orozco y Esther González, 1997, Los mexicanos en Estados Unidos, Sistemas Técnicos de Edición, México.

Duquette-Rury, Lauren y Xóchitl Bada, 2013, "Continuity and Change in Mexican Migrant Hometown Associations: Evidence from New Survey Research”, en Migraciones Internacionales, vol. 7, número especial 1.

Escala-Rabadán, Luis, 2004, "Migración y formas organizativas en los Estados Unidos: los clubes y federaciones de migrantes mexicanos en California", en Guillaume Lanly y M. Basilia Valenzuela (comps.), Clubes de migrantes oriundos mexicanos en los Estados Unidos: la politica transnacional de la nueva sociedad civil migrante, Universidad de Guadalajara, Zapopan.

__. Xóchitl Bada y Gaspar Rivera-Salgado, 2006, "Mexican Migrant Civic and Political Participation in the U. S.: The Case of Hometown Associations in Los Angeles and Chicago", en Norteamérica. Revista Académica del CISAN-UNAM, año 1, núm. 2.

__ Gaspar Rivera-Salgado y Rigoberto Rodríguez, 2011, "Is More Necessarily Better? Leadership and Organizational Development of Migrant Hometown Associations in Los Angeles, California”, en Migraciones Internacionales, vol. 6, núm. 2.

Esparza, Armando, 2000, “Evaluación del Programa 'Tres por Uno”, en Miguel Moctezuma (comp.), Memorias del Foro Sivilla-Fundación Produce sobre Temas de Migración, Ediciones y Publicaciones Gonber, Zacatecas.

Espinosa, Víctor M., 1999, The Federation of Michoacán Clubs in Illinois. The Chicago-Michoacán Project Report, Heartland Alliance for Human Needs and Human Rights, Chicago.

Fernández de Castro, Rafael, Rodolfo García Zamora y Ana Vila Freyer (coords.), 2006, El Programa 3 × 1 para migrantes: ¿iprimera política transnacional en México?, Instituto Tecnológico Autónomo de México, Universidad Autónoma de Zacatecas, Miguel Ángel Porrúa, México.

Fox, Jonathan, 2005a, "Mapping Mexican Migrant Civil Society", ponencia, "Mexican Migrant Social and Civic Participation”, Woodrow Wilson Center for Scholars, noviembre, en línea: <http://www.wilsoncenter.org/sites/default/files/Fox\%20-\%20Mapping\%20Mex\%20 Migrant\%20Civil\%20Society.pdf>.

—_, 2005b, "El sur en el norte: la emergente sociedad civil migrante", ponencia, Coloquio Internacional "Imperio y Resistencias", 4 de octubre, México.

__ 2005c, “Unpacking 'Transnational Citizenship”, en Annual Review of Political Science, vol. 8, pp. 171-201.

__ y Gaspar Rivera-Salgado (coords.), 2004, Indígenas migrantes mexicanos en los Estados Unidos, Miguel Ángel Porrúa, Universidad Autónoma de Zacatecas, Universidad de California, Santa Cruz y Zacatecas.

García, Juan Ramón, 1996, Mexicans in the Midwest, 1900-1932, University of Arizona Press, Tucson.

García Zamora, Rodolfo, 2000, Agricultura, migración y desarrollo regional, Universidad Autónoma de Zacatecas, Zacatecas. 
—_, 2001, Migración internacional y desarrollo local: los actores sociales y el reto de las nuevas políticas públicas en México, Universidad Autónoma de Zacatecas, Zacatecas.

—_, 2012, Crisis, migración y desarrollo. Los actores sociales y el reto de las nuevas políticas públicas en México, Universidad Autónoma de Zacatecas, Zacatecas.

Goldring, Luin, 1995, "Blurring Borders: Constructing Transnational Community Process of México-U. S. Migration”, en Research in Community Sociology, núm. 6, pp. 69-104.

_ American Research Review, vol. 37, núm. 3, pp. 55-99.

González Gutiérrez, Carlos, 1993, "Mexican Diaspora in California: Limits and Possibilities of the Mexican Government”, en Abraham Lowenthal y Katrina Burgess (eds.), The California-Mexico Connection, Stanford University Press, Stanford.

, 1995, "La organización de los inmigrantes mexicanos en Los Ángeles: la lealtad de los oriundos", en Revista Mexicana de Política Exterior, núm. 46, pp. 59-101.

(coord.), 2006, Relaciones Estado-diáspora: aproximaciones desde cuatro continentes, Secretaría de Relaciones Exteriores, Miguel Ángel Porrúa, México.

González Zepeda, Carlos A., 2012, "Isomorfismo institucional y promoción del desarrollo local en asociaciones de migrantes michoacanos en Los Ángeles, California”, tesis de maestría en desarrollo regional, El Colegio de la Frontera Norte, Tijuana.

Gordon, Dan, 2005, "Local Heroes. How Mexican Immigrants Have Forged Complex Government Partnerships to Improve Living Conditions Back Home", en Stanford Social Innovation Review, vol. 3, núm. 3, pp. 51-53.

Hecht, Peter, 2005, "A Drive for Clout: Community Groups Representing Mexican Migrants Form a Confederation to Influence Public Policy in California", en The Sacramento Bee, 29 de agosto, p. A1.

Ímaz Bayona, Cecilia, 2006, La nación mexicana transfronteras. Impactos sociopolíticos en México de la emigración a Estados Unidos, Facultad de Ciencias Políticas y Sociales-Universidad Nacional Autónoma de México, México.

Lanly, Guillaume y Basilia Valenzuela, 2004, “Introducción”, en Guillaume Lanly y M. Basilia Valenzuela (comps.), Clubes de migrantes oriundos mexicanos en los Estados Unidos: la política transnacional de la nueva sociedad civil migrante, Universidad de Guadalajara, Zapopan.

Leal, Claudia y Luis Escala-Rabadán, 2011, "Formas organizativas migrantes y gobernanza local en México”, en Cofactor, vol. 2, núm. 3.

Light, Ivan, 2006, Deflecting Immigration: Networks, Markets, and Regulation in Los Angeles, Russell Sage Foundation, Nueva York.

Massey, Douglas et al., 1987, Return to Aztlán. The Social Process of International Migration from Western Mexico, University of California Press, Berkeley.

Mestries, Francis, 1998, “Tradición migratoria y organización comunitaria: el caso de Zacatecas”, en René Zenteno (ed.), Pob/ación, desarrollo y globalización, Sociedad Mexicana de Demografía, El Colegio de la Frontera Norte, México.

Mexican American Legal Defense and Educational Fund (MALDEF), 2005, "MALDEF Launches Immigrant Leadership Program", comunicado de prensa, 18 de mayo.

Mines, Richard, 1981, Developing a Community Tradition of Migration: A Field Study in Rural Zacatecas, Mexico and California Settlement Areas, Center for U. S.-Mexican Studies-University of California, San Diego, La Jolla.

Moctezuma, Miguel, 2011, La transnacionalidad de los sujetos. Dimensiones, metodologías y prácticas convergentes de los inmigrantes mexicanos en Estados Unidos, Universidad Autónoma de Zacatecas, Miguel Ángel Porrúa, México.

Morán, Rodolfo, 1998, “Los sentidos de la transición: migrantes internacionales y cultura regional”, tesis de doctorado, El Colegio de la Frontera Norte, Tijuana.

Moya, José C., 2005, "Immigrants and Associations: A Global and Historical Perspective”, en Journal of Ethnic and Migration Studies, vol. 31 , núm. 5. Orozco, Manuel y Katherine Welle, 2005, "Clubes de migrantes y desarrollo: pertenencia, concordancia, sustentabilidad y replicabilidad", en Barbara Merz (comp.), Nuevas pautas para México. Observaciones sobre remesas, donaciones filantrópicas y desarrollo equitativo, Global Equity Initiative-Harvard University, Cambridge.

Passel, Jeffrey S., 2005, Estimates of the Size and Characteristics of the Undocumented Population, Pew Hispanic Center, Washington, D. C. Putnam, Robert D., 2000, Bowling Alone. The Collapse and Revival of American Community, Simon and Schuster, Nueva York.

Ramakrishnan, S. Karthick e Irene Bloemraad, 2008, "Introduction: Civic and Political Inequalities", en S. Karthick Ramkrishnan e Irene Bloemraad (eds.), Civic Hopes and Political Realities. Immigrants, Community Organizations, and Political Engagement, Russell Sage Foundation, Nueva York. 
Rivera, Alex, 2003, The Sixth Section, video documental, kPBs-Point of View, Fundación MacArthur, Fundación Rockefeller, Nueva York. Rivera-Salgado, Gaspar, 1999, "Binational Organizations of Mexican Migrants in the United States”, en Social Justice, vol. 26, núm. 3.

y Luis Escala-Rabadán, 2008, "Migration, Collective Remittances, and Development: Mexican Migrant Associations in the United States", en Ton van Naerssen, Ernst Spaan y Annelies Zoomers (comps.), Global Migration and Development, Routledge, Nueva York.

Santa Ana, Otto, 2002, Brown Tide Rising. Metaphors of Latinos in Contemporary American Public Discourse, University of Texas Press, Austin.

Secretaría de Relaciones Exteriores (SRE), 2009, “Directorio de oriundos en los Estados Unidos”, Secretaría de Relaciones Exteriores, México.

__, 2012, "Programa para las Comunidades Mexicanas en el Exterior", Secretaría de Relaciones Exteriores, México.

Shannon, Amy, 2006, "Las organizaciones transnacionales como agentes del desarrollo local. Retos y oportunidades del Programa $3 \times 1$ para Migrantes”, en Rafael Fernández de Castro, Rodolfo García Zamora y Ana Vila Freyer (comps.), El Programa $3 \times 1$ para migrantes: ¿primera política transnacional en México?, Instituto Tecnológico Autónomo de México, Universidad Autónoma de Zacatecas, Miguel Ángel Porrúa, México.

Smith, Robert, 2001, "Comparing Local-Level Swedish and Mexican Transnational Life: An Essay in Historical Retrieval”, en L. Pries (comp.), New Transnational Social Spaces. International Migration and Transnational Companies in the Early Twenty-First Century, Routledge, Londres.

Somerville, Will, Jamie Durana y Aaron Matteo Terrazas, 2008, “Hometown Associations: An Untapped Resource for Immigrant Integration?”, en Insight, julio, Migration Policy Institute.

Soyer, Daniel, 1997, Jewish Immigrant Associations and American Identity in New York, 1880-1939, Harvard University Press, Cambridge.

Taylor, Lawrence, 1997, “Las fiestas patrias y la preservación de la identidad cultural mexicana en California: una visión histórica”, en Frontera Norte, vol. 9, núm. 18.

Thompson, Ginger, 2005, “Mexico's Migrants Profit from Dollars Sent Home”, en The New York Times, 23 de febrero.

United Nations Development Programme (UNDP), 2006, "The Potential Role of Remittances in Achieving the Millennium Development Goals. An Exploration", Roundtable on Remittances and the Millennium Development Goals, United Nations Development Programme, Nueva York.

Vázquez Mota, Josefina, 2005, "El Programa Iniciativa Ciudadana 3 × 1. Un instrumento para respaldar la inversión social de los inmigrantes mexicanos”, en Foreign Affairs en Español, vol. 5, núm. 3.

Vázquez Valenzuela, David Adán, 2012, "Mirando atrás: la comunidad mexicana y mexicoamericana de Los Ángeles ante la Revolución Mexicana. Su participación en el floresmagonismo, 1905-1911", tesis de maestría, Instituto de Investigaciones Históricas Dr. José María Luis Mora, México.

Viramontes, Celia, 2008, "Civic Engagement across Borders: Mexicans in Southern California”, en S. Karthick Ramkrishnan e Irene Bloemraad (eds.), Civic Hopes and Political Realities. Immigrants, Community Organizations, and Political Engagement, Russell Sage Foundation, Nueva York.

Waldinger, Roger, 2013, "Más allá del transnacionalismo: una perspectiva alternativa de la conexión de los inmigrantes con su país de origen", en Migraciones Internacionales, vol. 7, número especial 1.

Wides, Laura, 2004, “Immigrant Mexican Philanthropic Groups Turn toward Politics”, en Union-Tribune San Diego, 19 de enero.

Yrízar, Guillermo y Rafael Alarcón, 2010, "Emigration Policy and State Governments in Mexico", en Migraciones Internacionales, vol. 5, núm. 4.

Zabin, Carol et al., 1993, Mixtec Migrants in California Agriculture: A New Cycle of Poverty, California Institute for Rural Studies, Davis.

Zabin, Carol y Luis Escala-Rabadán, 2002, "From Civic Association to Political Participation: Mexican Hometown Associations and Mexican Immigrant Political Empowerment in Los Angeles", en Frontera Norte, vol. 14, núm 27. 